\title{
SOME RECENT OBSERVATIONS OF \\ OCCULTATIONS BY THE MOON
}

\author{
NATHANIEL M. WHITE \\ Lowell Observatory, Flagstaff, Arizona 86001, U.S.A.
}

\begin{abstract}
A program for the measurement of diffraction patterns resulting from the lunar occultations of stars was begun at the Lowell Observatory by Rakos (1964), resumed by him in 1967 and continued by Pettauer through the summer of 1969. The expected results were accurate timing of occultations and hence accurate position measurements, the discovery of close double stars, and the determination of stellar diameters. The author is continuing the program using new equipment designed by R. E. Nather of the University of Texas.

The equipment was built at the Lowell Observatory and put into operation on its new 42-in. reflector in March, 1970.
\end{abstract}

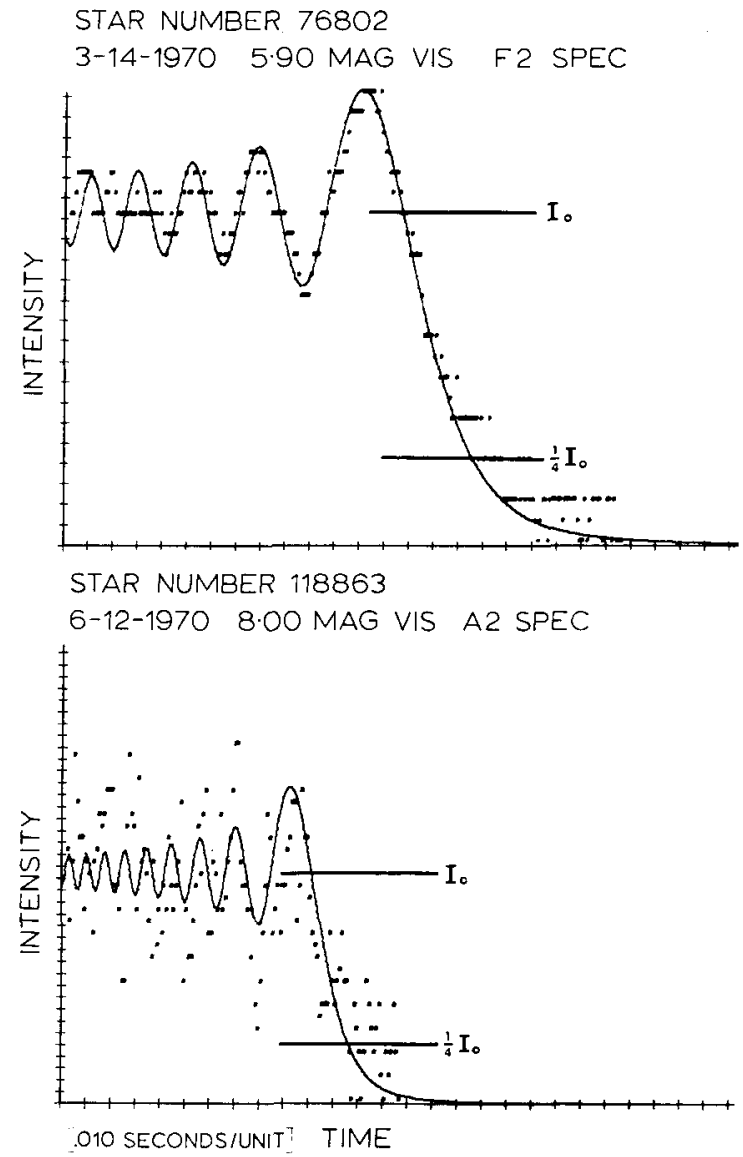

Fig. 1. 
The most important operational characteristics of the occultation equipment are:

(1) Selective amplification of the star signal above the sky for greater contrast.

(2) Impersonal, electronic sensing of the occultation occurrence.

(3) Digitized output which is recorded on punched paper tape and strip chart recorder.

With this equipment about 60 occultations have been observed and more than 50 occultation times have been determined. The classical theory of light diffraction by a straight-edge shows that the time of geometrical occultation occurs when the intensity has dropped to $25 \%$ of the mean intensity before the occultation.

Figure 1 demonstrates the possible timing accuracy and its dependence on noise. The position of geometrical occultation is well defined to within a few milliseconds on the time scale of the low-noise pattern. The same position on the noisy tracing could be in error by $10 \mathrm{msec}$ or more. The noise appears to be more dependent on direct atmospheric effects than on the star's apparent brightness.

No convincing diameter measurements have been made so far. However, Figure 2 shows the kind of results that might be expected. Four preliminary representations

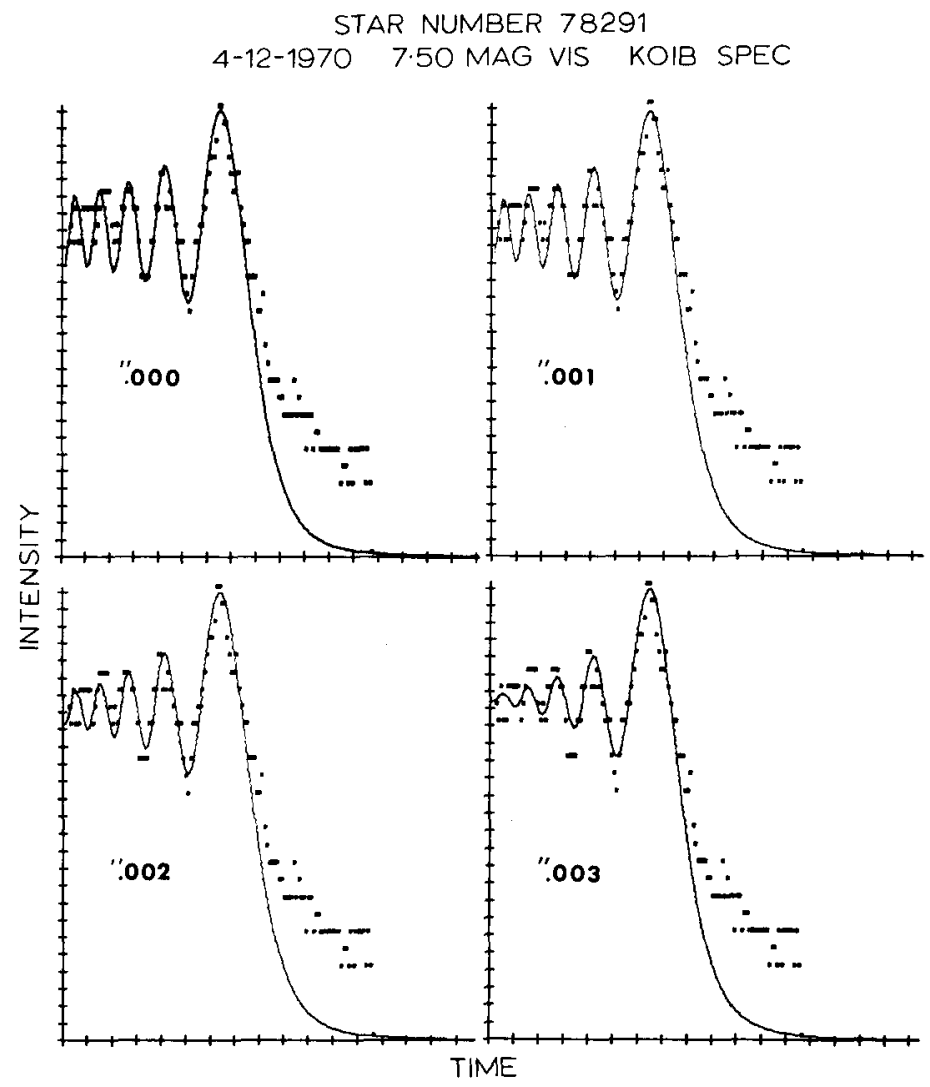

Fig. 2. 
of an observed pattern produced by a KOIb star are shown. From its apparent magnitude and temperature, this star is expected to have a diameter somewhat less than $0.001 \mathrm{arcsec}$. On close inspection it can be seen that the best fit occurs for a star that lies between a point source and a source of 0.001 arcsec in diameter. A pattern for a 0.003 arcsec diameter star definitely does not fit the observations.

Deconvolution methods of analyzing the data for diameters and duplicity are now being worked on, but have not yet been applied to our recent data.

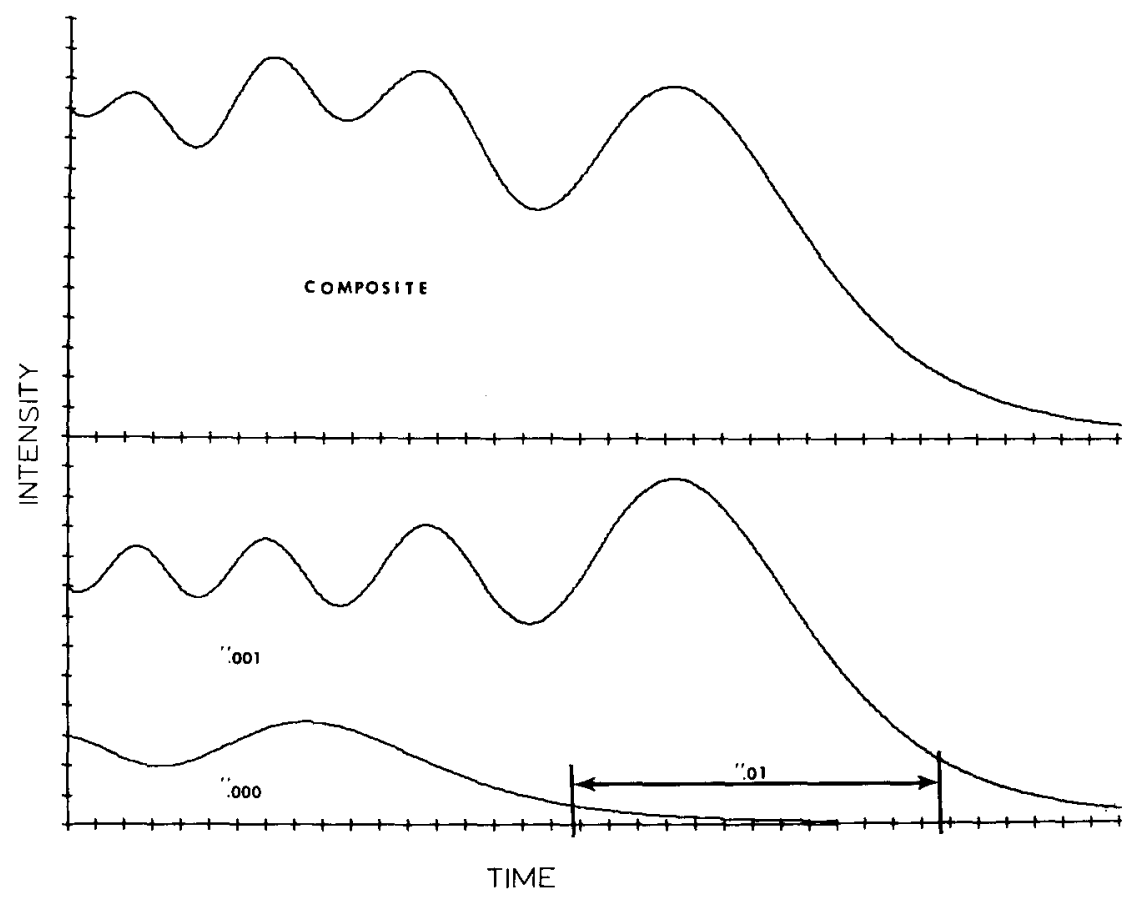

Fig. 3.

Duplicity of stars unresolved by any other technique can be detected from their diffraction patterns. A single observation yields the relative magnitudes and apparent projected separation of the components of the double star. For example, by summing two patterns corresponding to diameters of 0.000 and 0.001 arcsec, separated by $0.010 \mathrm{arcsec}$, and of relative intensities 3.4 and $1.0\left(\Delta \mathrm{mag}=1.3 \mathrm{~m}_{\mathrm{vis}}\right)$, the composite pattern shown in Figure 3 is obtained. This pattern closely resembles that observed for a G7III star of apparent visual magnitude 5.60 as shown in Figure 4.

While it is also possible that this peculiar pattern might be caused by lunar limb irregularities, this can neither be confirmed nor ruled out on the basis of a single observation at only one location. It is, however, of interest to note that existing radial velocity measures of this star indicate the radial velocity to be variable with a suggested period of less than one year. This is completely compatible with the assumption 


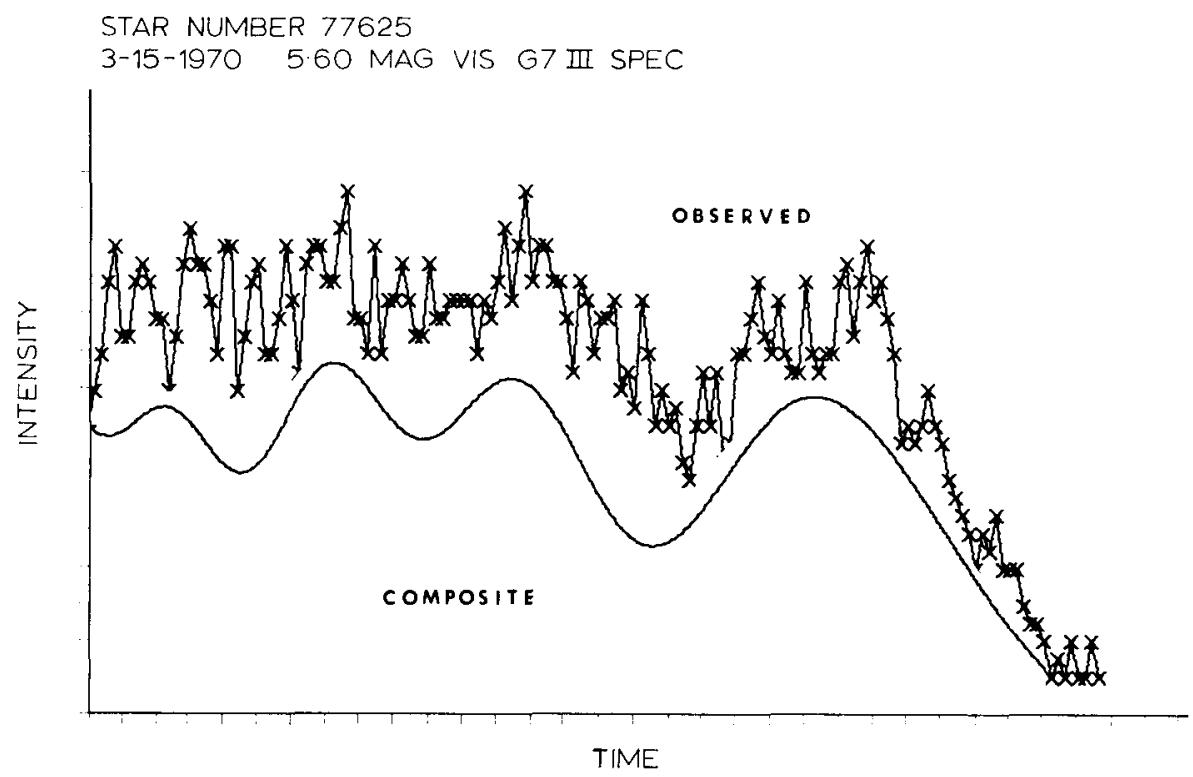

Fig. 4.

that the observed pattern is caused by a binary of a separation of up to 0.018 arcsec and having an $\mathrm{A} 5 \mathrm{~V}$ star as its second component. On the basis of this evidence, this star has already been placed on H. A. Abt's radial velocity program at Kitt Peak National Observatory.

While the patterns discussed represent only a few examples of results thus far obtained from this program, they demonstrate, nevertheless, the potential of occultation measurements as a powerful and promising observational technique.

Plans are now being made to set aside the Lowell 30-in. reflector for the sole purpose of occultation measurements. This is expected to increase both the quantity and quality of the observations because of the telescope's exclusive availability for occultation observations and because of the resulting possibility of customizing it entirely for this purpose.

A PDP-11 on-line computer is expected to be in operation at Lowell Observatory's 42-in. telescope by the fall of 1970 , and will be available for use in occultation measurements. For the most interesting stars an effort will be made to carry out simultaneous observations with the 42-in. and 30-in. telescopes. Moreover, cooperation with other observatories, which may be of great importance to the ultimate success of any occultation program, will be actively sought and offered by Lowell Observatory.

\section{References}

Nather, R. E.: 1970, Astron. J. 75, 583.

Rakos, K. D.: 1964, Astron. J. 69, 556 (abstract). 